\title{
Conditions for Phase Locking and Dephasing of Autoresonant Pumping
}

\section{O. M. Kiselev}

We study the asymptotic behavior of nonlinear oscillators under an external driver with slowly changing frequency and amplitude. As a result, we obtain formulas for properties of the amplitude and frequency of the driver when the autoresonant behavior of the nonlinear oscillator is observed. Also, we find the measure of autoresonant asymptotic behaviors for such a driven nonlinear oscillator.

Keywords: nonlinear oscillator, autoresonance, perturbations

\section{Introduction}

An autoresonance gives an opportunity to excite a nonlinear oscillator by a small oscillating force. Phase-locking was first proposed for pumping of energy into beams of particles in a synchrotron $[1,2]$. A key condition for autoresonant excitation is phase-locking, when the difference between the phase of the perturbing force and the phase of the oscillator is practically constant. Phase-locking is a well-known phenomenon for a wide class of nonlinear oscillators. A connection between autoresonance and phase-locking was mentioned, for example, in [3]. Now phase-locking and autoresonance are considered as general ways to change the energy of nonlinear oscillators in the general case, from planetary motion [4] to low-temperature techniques [5].

From a mathematical point of view, a typical equation with autoresonant solutions is that of a nonlinear oscillator with a small oscillating perturbation:

$$
u^{\prime \prime}+G(u)=\varepsilon F \cos (\Phi(t, \varepsilon)) .
$$

Here $G$ is a nonlinear function such that $G(u) \sim u+g_{3} u^{3}$, as $u \rightarrow 0, \varepsilon$ is a small positive parameter, and $F$ is a nonoscillating function of $t$ and $\epsilon$. Without loss of generality one can see two types of examples. The first is an equation for the pendulum with an external driver:

$$
\frac{d^{2} u}{d t^{2}}+\sin (u)=\epsilon F(t, \epsilon) \cos (t-s(t, \epsilon))
$$

Received May 07, 2019

Accepted September 20, 2019

Oleg M. Kiselev

ok@ufanet.ru

Institute of Mathematics with Computing Centre

ul. Chernyshevskogo 112, Ufa, 450008 Russia

RUSSIAN JOURNAL OF NONLINEAR DYNAMICS, 2019, 15(3), 381-394 
the other is a Duffing oscillator:

$$
\frac{d^{2} u}{d t^{2}}+u-\frac{u^{3}}{6}=\epsilon F(t, \epsilon) \cos (t-s(t, \epsilon)) .
$$

Here $0<\epsilon \ll 1, F(t, \epsilon)$ is the amplitude of perturbation, $s(t, \epsilon)$ is the phase shift of the resonant frequency of perturbation. An additional condition on the shift is slow dependence on $t$. It is convenient to apply: $s^{\prime}=O\left(\epsilon^{2 / 3}\right)$, where $s^{\prime}(t, \epsilon)$ is such that $s^{\prime \prime}(t, \epsilon)=o\left(s^{\prime}\right)$.

The autoresonance condition is a thin balance between the amplitude and the phase of the perturbation force [4]. One can see energies for different trajectories in Fig. 1. Our goal is to explain that the growing autoresonance solutions exist even for perturbations with decreasing amplitude, see trajectory 2 in Fig. 1 . Of course, one can see that this observation is in no contradiction with earlier results for Eq. (4) as $s^{\prime} \equiv \tau$ and $\mathcal{F}=$ const if one makes an attempt to widely extrapolate consequences on the class of Eq. (4) with nonconstant coefficients. In fact, there is a general theory for systems with slowly varying coefficients, see [23]. But the autoresonant growth of nonlinear oscillations in the presence of decreasing pumping does not seem to have been mentioned before in this field.

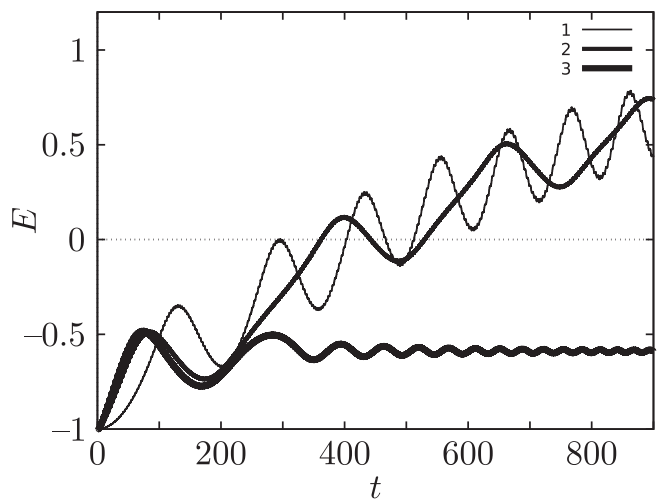

Fig. 1. The energy of a perturbed pendulum (2): $E(t)=\left(u^{\prime}\right)^{2} / 2-\cos (u)$, for different perturbation forces. On the first curve $F=8$, on the second $F=8 \tau^{-1 / 2}$ and on the third $F=8 \tau^{-4 / 7}$. Here $\tau=\epsilon^{2 / 3} t$, $s=\tau^{2} / 2, \epsilon=1 / 800$. One can see the growth of energy on curves 1 and 2 and the stabilization of energy on curve 3 .

A primary resonance equation defines the amplitude of a small oscillation of order $O(\sqrt[3]{\varepsilon})$. This equation has the form

$$
i \frac{d \Psi}{d \tau}+\left(s^{\prime}(\tau)-|\Psi|^{2}\right) \Psi=\mathcal{F}(\tau) .
$$

Usually this equation is considered for special values of coefficients: $s^{\prime} \equiv \tau$ and $\mathcal{F}(\tau)=$ const. In such a case the solution has two types of two-parametric solutions. The first is a bounded solution with a quadratic growth of frequency. The second is a solution growing as $\sqrt{\tau}$. This one is called an autoresonant solution.

One can connect the solutions of (4) and solutions of nonlinear oscillator (1). The autoresonant solutions of (4) define nonlinear oscillations of (1) with growing amplitude. Numerous applications of (1) can be found, for example, in [4]. Threshold values of parameters for autoresonance were discussed in [4] and [6]. An asymptotic analysis of the threshold value for the external force for two coupled oscillators was made in [7]. Asymptotic behavior for solutions over long times was considered in the review [8]. 
The asymptotic behavior of $s(\tau)$ and $\mathcal{F}(\tau)$ in $(4)$ is important for the autoresonant growth. Below we consider the coefficients of algebraic type: $s^{\prime}(\tau)=\lambda^{2} \tau^{2 a}$ and $\mathcal{F}(\tau)=f \tau^{b}$, where $a>0$, $b, \lambda, f \in \mathbf{R}$. Different curves for the energy of the perturbed pendulum are shown in Fig. 1.

In this work we have found intervals for values of the amplitude and frequency of the driver which lead to autoresonant behavior of the solutions. In particular, we have found that the stable autoresonant growth exists for decreasing amplitudes of the driver. We study the stability of autoresonant behavior and show parameter values for stability, see also [9]. This result is more general than that of the earlier studies of autoresonance for the special case $\mathcal{F} \equiv$ const and $a=1 / 2$ which are discussed in [10-12].

Here we obtain results on the phase locking and dephasing of trajectories concerning the autoresonance area. We recall earlier results in this field $[13,14]$ and a more complete asymptotic approach in the special case [15]. In particular, Eq. (4) in the special case where $s \equiv \tau$ and $\mathcal{F} \equiv$ const is considered in [13] and partially in [14] and [15]. In [13] a change of action during a separatrix crossing was found. Some of the results of [14] show that the Painlevé transcendents are helpful in describing the asymptotics of the solution near a separatrix. In [15], full asymptotic expansions for the solution which crossed the separatrix were obtained.

Since the work of Poincaré [16] a lot of approaches using Hamiltonian theory have been proposed to study nonlinear oscillators with a small parameter. But in this work we consider asymptotic behaviors of solutions at large times in the special case where the growth of the coefficients in the equations under study is more important than the dependence on a small parameter. Therefore, the asymptotic methods developed by Krylov and Bogolyubov [17] with approaches for large time asymptotic expansions in the spirit of the books [18] and [19] appear more convenient for calculations.

A review of Hamiltonian approaches for perturbation analysis of equations like (1) and averaging theory can be found in [20-23] and references therein.

This paper is structured as follows. In Section 1 we present a well-known approach to deriving an equation for primary autoresonance for nonlinear oscillators under the external driver. In Section 2 we find necessary conditions for parameters of Eq. (4) when growing autoresonant behavior may exist. In Section 3 we derive an equation for autoresonance and obtain a slowly varying autoresonant asymptotic expansion. In Section 4 we show an equation which defines a capture into autoresonance, calculate a gap between separatrices and get a measure for trajectories which pass through this gap. In Section 5 we obtain asymptotic behavior of a measure for autoresonant solutions of a nonlinear oscillator.

\section{Derivation of an equation for autoresonance}

The equation for primary autoresonance defines a long temporary evolution of the amplitude for fast oscillations of a nonlinear oscillator under resonant perturbation with a slow shift of the perturbation frequency. As an example, one can consider the equation for a perturbed pendulum (2) or the equation for a Duffing oscillator (3). One can find solutions to these equations for small values of $u$ in the form of a fast oscillating function with a slow modulated amplitude:

$$
u=\sqrt[3]{\epsilon} A e^{i(t-s)}+\text { c.c. }
$$

Here $A=A(\tau)$ and $\tau=\epsilon^{2 / 3} t$ is slow time. Substituting this formula into (2), we get

$$
\epsilon\left(2 i A^{\prime}+2 s^{\prime} A-\frac{1}{2}|A|^{2} A\right) e^{i(t-s)}+o(\epsilon)=\epsilon \frac{1}{2} F e^{i(t-s)} .
$$


Then in primary order in $\epsilon$ one gets an equation for the slowly varying amplitude $A(\tau)$

$$
2 i A^{\prime}+2 s^{\prime} A-\frac{1}{2}|A|^{2} A=\frac{1}{2} F .
$$

Let us make the change of variables: $A=2 \Psi$ and $F=8 \mathcal{F}$. Then we get equation (4) for the complex-valued function $\psi$.

Such an approach for derivation of the autoresonant equation follows the averaging method [17]. This approach for autoresonance is well-known, see, e.g., [24, 25]. A detailed review for autoresonance and its connection with nonlinear oscillators are presented in [9]. An asymptotic analysis of autoresonant growth for a nonlinear oscillator from small values up to $O(1)$ is developed in [26].

\section{Necessary values of frequency and the amplitude for phase locking}

Let us substitute coefficients into (4):

$$
i \frac{d \Psi}{d \tau}+\left(\lambda^{2} \tau^{2 a}-|\Psi|^{2}\right) \Psi=f \tau^{b}
$$

Equation (8) is in fact a nonautonomous Hamiltonian system with one degree of freedom. Let us denote $\Psi=x+i y$, where $x(\tau)$ and $y(\tau)$ are real functions. Then Eq. (8) yields

$$
\frac{d x}{d \tau}=y^{3}+x^{2} y-\lambda^{2} \tau^{2 a} y, \quad \frac{d y}{d \tau}=-x y^{2}-x^{3}+\lambda^{2} \tau^{2 a} x-f \tau^{b} .
$$

Then the Hamiltonian becomes

$$
H(x, y, \tau)=-\frac{\lambda^{2} \tau^{2 a}}{2}\left(x^{2}+y^{2}\right)+\frac{1}{4}\left(x^{2}+y^{2}\right)^{2}+f \tau^{b} x .
$$

The equation for $x$ and $y$ can be written as

$$
\frac{d x}{d \tau}=\frac{\partial H}{\partial y}, \quad \frac{d y}{d \tau}=-\frac{\partial H}{\partial x} .
$$

The amplitude of the leading-order term of autoresonant asymptotic behavior for the solution as $\tau \rightarrow \infty$ is defined from the following equation:

$$
\left(\lambda^{2} \tau^{2 a}-|\Psi|^{2}\right)=o\left(\tau^{2 a}\right) .
$$

Therefore, for autoresonant solutions one gets the condition $|\Psi| \sim \lambda \tau^{a}$.

It is convenient to write Eq. (8) in such a form that the growing part of the solution can be singled out explicitly. Let us make the change of variable:

$$
\Psi=\lambda \tau^{a} \psi \exp \left(i \lambda^{2} \frac{\tau^{2 a+1}}{2 a+1}\right) .
$$

This yields

$$
i \tau^{-2 a} \lambda^{-2} \psi^{\prime}-|\psi|^{2} \psi=\frac{f \tau^{b}}{\lambda^{3} \tau^{3 a}} \exp \left(-i \lambda^{2} \frac{\tau^{2 a+1}}{2 a+1}\right)-i \frac{a \psi}{\lambda^{2} \tau^{2 a+1}} .
$$


Here it is convenient to use a new variable: $\theta=\lambda^{2} \tau^{2 a+1} /(2 a+1)$ and assume that $\psi=\psi(\theta)$. Then the equation for primary resonance has the form

$$
i \frac{d \psi}{d \theta}-|\psi|^{2} \psi=\frac{(2 a+1)^{\frac{b-3 a}{2 a+1}}}{\lambda^{\frac{3+2 b}{2 a+1}}} \frac{f \exp (-i \theta)}{\theta^{\frac{3 a-b}{2 a+1}}}-\frac{i a}{2 a+1} \frac{\psi}{\theta} .
$$

Let us define:

$$
\nu=\frac{3 a-b}{2 a+1}, \quad h=f(2 a+1)^{-\nu} \lambda^{-\frac{2 b+3}{2 a+1}}, \quad q=\frac{a}{2 a+1} .
$$

As a result, we obtain an equation for $\psi$ in the form

$$
i \frac{d \psi}{d \theta}-|\psi|^{2} \psi=\frac{h e^{-i \theta}}{\theta^{\nu}}-i \frac{q \psi}{\theta} .
$$

If $\nu>0$, then the right-hand side of the equation is small as $\theta \rightarrow \infty$. To study the asymptotic behavior of solutions for large $\theta$, we obtain an equation for $|\psi|^{2}$. For this we use equations for $\psi$ and complex conjugation $\bar{\psi}$ :

$$
\begin{aligned}
& \frac{d \psi}{d \theta}=-i|\psi|^{2} \psi-i \frac{h e^{-i \theta}}{\theta^{\nu}}-\frac{q}{\theta} \psi, \\
& \frac{d \bar{\psi}}{d \theta}=i|\psi|^{2} \bar{\psi}+i \frac{h e^{i \theta}}{\theta^{\nu}}-\frac{q}{\theta} \bar{\psi} .
\end{aligned}
$$

Let us differentiate $|\psi|^{2}$ using these equations:

$$
\frac{d|\psi|^{2}}{d \theta}=\frac{d \psi}{d \theta} \bar{\psi}+\psi \frac{d \bar{\psi}}{d \theta}=\frac{h}{\theta^{\nu}}\left(i \psi e^{i \theta}-i \bar{\psi} e^{-i \theta}\right)-2 \frac{q}{\theta}|\psi|^{2} .
$$

Here we define $\psi=R e^{i \phi}$, where $R=R(\theta)$ and $\phi=\phi(\theta)$ are real-valued functions. Then Eq. (11) can be rewritten in the form of a differential equation for $R$ :

$$
\frac{d R}{d \theta}=-\frac{h}{\theta^{\nu}} \sin (\theta+\phi)-\frac{q}{\theta} r .
$$

Let us find the maximum of order for the growth of $R$. To do so, we estimate: $\sin (\theta+\phi)=-1$. Let us define a majorant $\tilde{R}>R$. For this majorant we obtain the equation

$$
\frac{d \tilde{R}}{d \theta}=\frac{h}{\theta^{\nu}}-\frac{q}{\theta} \tilde{R}
$$

Then

$$
\tilde{R}=\frac{\theta^{1-\nu}}{1+q-\nu}+C \theta^{-q}, \quad C \in \mathbb{R} .
$$

Using the formula for $q$, we get $0<q<1 / 2$ when $a>0$. The majorant does not decrease if $\nu<1$. This gives $0<\nu<1$. Therefore, the parameters $a$ and $b$ are

$$
0<\frac{3 a-b}{2 a+1}<1
$$

Theorem 1. The necessary conditions for the existence of a nondecreasing solution are

$$
a>0, \quad a-1 \leqslant b \leqslant 3 a .
$$

For the linear chirp-rate the frequency $s$ is defined by $a=1 / 2$. Then the necessary conditions for autoresonance for the amplitude of the driver are $\tilde{f}_{1} \tau^{-1 / 2} \leqslant f \leqslant \hat{f}_{1} \tau^{3 / 2}$, where $\tilde{f}_{1}, \hat{f}_{1}$ are some constants. 


\section{Asymptotic substitution}

In this section we study the behavior of $\psi$ which is defined in (9). Here we assume that $\psi$ is bounded.

To find the asymptotic behavior for solutions of (10) as $\theta \rightarrow \infty$, one can write the equation for the amplitude and phase for the function $\psi$. Let us make the change of variable:

$$
\psi=(1+\rho) e^{i(\alpha-\theta)},
$$

where $\rho, \alpha$ are real-valued functions of $\theta$.

Let us substitute these formulas into (10), multiply the left- and right-hand sides of the equation by $e^{-i(\alpha-\theta)}$. Then we obtain a system of equations for real and imaginary parts of (10):

$$
\begin{gathered}
\alpha^{\prime}+\frac{h}{\theta^{\nu}} \frac{\cos (\alpha)}{(1+\rho)}+\rho^{2}+2 \rho=0, \\
\rho^{\prime}+\frac{h}{\theta^{\nu}} \sin (\alpha)+\frac{q}{\theta} \rho+\frac{q}{\theta}=0 .
\end{gathered}
$$

Here one should find values of coefficients of this system for which the necessary conditions for the existence of autoresonant solutions are valid: $0<\nu<1$.

To construct a solution with small $\rho$, it is convenient to rewrite: $\sigma(\theta)=\sin (\alpha)$, then $\sigma^{\prime}=\alpha^{\prime} \cos (\alpha)$. The system can be written as

$$
\begin{aligned}
\sigma^{\prime}+\frac{h}{\theta^{\nu}} \frac{1-\sigma^{2}}{(1+\rho)}+\left(\rho^{2}+2 \rho\right) \kappa \sqrt{1-\sigma^{2}} & =0, \\
\rho^{\prime}+\frac{h}{\theta^{\nu}} \sigma+\frac{q}{\theta} \rho+\frac{q}{\theta} & =0 .
\end{aligned}
$$

Here $\kappa=\operatorname{sign}(\cos (\alpha))$.

\subsection{Slowly varying asymptotic expansions}

When $\theta \rightarrow \infty$ the system of equations has two small parameters. There are $\theta^{\nu-1}$ and $\theta^{-\nu}$. Asymptotic expansions for formal series can be obtained using a two-scaling method, where $\zeta=\theta^{\nu}, \xi=\theta^{1-\nu}$,

$$
\frac{d}{d \theta}=\frac{d \zeta}{d \theta} \frac{\partial}{\xi \partial \zeta}+\frac{d \xi}{d \theta} \frac{\partial}{\zeta \partial \xi}=\frac{\nu}{\xi} \frac{\partial}{\partial \zeta}+\frac{1-\nu}{\zeta} \frac{\partial}{\partial \xi} .
$$

The system of equations can be rewritten as

$$
\begin{aligned}
\frac{\nu}{\xi} \frac{\partial \sigma}{\partial \zeta}+\frac{1-\nu}{\zeta} \frac{\partial \sigma}{\partial \xi}+\frac{h}{\theta^{\nu}} \frac{1-\sigma^{2}}{(1+\rho)}+\left(\rho^{2}+2 \rho\right) \kappa \sqrt{1-\sigma^{2}} & =0, \\
\frac{\nu}{\xi} \frac{\partial \rho}{\partial \zeta}+\frac{1-\nu}{\zeta} \frac{\partial \rho}{\partial \xi}+\frac{h}{\zeta} \sigma+\frac{q}{\zeta \xi} \rho+\frac{q}{\zeta \xi} & =0 .
\end{aligned}
$$

The formal solution to this system as a series of negative powers of $\xi$ and $\zeta$ has the form

$$
\sigma \sim \sum_{j, k>0} \frac{\sigma_{j k}}{\xi^{j} \zeta^{k}}, \quad \rho \sim \sum_{j, k>0} \frac{\rho_{j k}}{\xi^{j} \zeta^{k}}, \quad \sigma_{j k}, \rho_{j k} \in \mathbf{R} .
$$

The coefficients of the asymptotic expansions for $\sigma_{j k}$ and $\rho_{j k}$ are obtained using recurrent equations for orders $\xi^{-j} \zeta^{-k}$. 
The leading-order terms have the form

$$
\sigma \sim-\kappa \frac{q}{h \xi}, \quad \rho \sim-\kappa \frac{h}{2 \zeta} .
$$

Using the system of equations for $\rho$ and $\alpha$ and the formulas for leading-order terms, we obtain

Theorem 2. The asymptotic behavior of the decreasing solution for $\left(\alpha_{*}, \rho_{*}\right)$ as $\theta \rightarrow \infty$ has the form

$$
\begin{array}{cl}
\alpha_{*} \sim \alpha_{0}+(-1)^{n+1} \frac{q}{h \theta^{1-\nu}}, & \rho_{*} \sim(-1)^{n+1} \frac{h}{2 \theta^{\nu}}-\frac{3 h^{2}}{8 \theta^{2 \nu}}, \\
\alpha_{0}=\pi n, \quad n=0,1 . &
\end{array}
$$

Here we obtain a pair of particular asymptotic solutions. These solutions do not contain parameters and these solutions can be considered to be slowly varying equilibria for the nonautonomous system of Eqs. (15).

\subsection{Fast oscillating asymptotic expansions}

In neighborhoods of slowly varying asymptotic solutions one can construct general solutions which contain arbitrary parameters. One can see such a trajectory in Fig. 2. Such solutions depend on the fast variable as $\theta \rightarrow \infty$. We separate the dependence on the fast variable using a system of equations in the form (13).

Let us define:

$$
\alpha=\alpha(S), \quad \rho=\theta^{-\nu / 2} r(S), \quad S=\frac{2}{2-\nu} \theta^{1-\nu / 2},
$$

where $S$ is a new fast variable and $r(S)$ represents the function $\rho$ in the new scale. This yields

$$
\begin{gathered}
\frac{d \alpha}{d S}=-2 r-\theta^{-\nu / 2}\left(r^{2}+\frac{h \cos (\alpha)}{1+\theta^{-\nu / 2} r}\right), \\
\frac{d r}{d S}=-h \sin (\alpha)-\theta^{-1+\nu} q-\theta^{-1+\nu / 2}\left(q-\frac{\nu}{2}\right) r .
\end{gathered}
$$
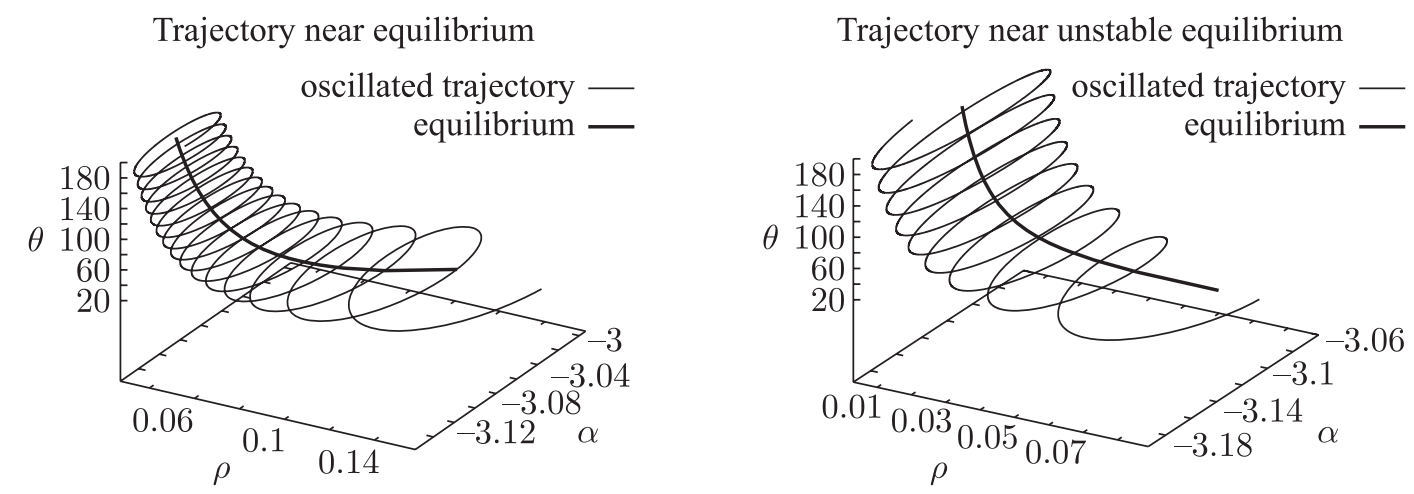

Fig. 2. Here one can see two oscillating trajectories near a slowly varying equilibrium. The trajectory was obtained by numerically solving the Cauchy problem for $\alpha=-\pi+q /\left(h \theta_{0}^{1-\nu}\right), \rho=h /\left(2 \theta_{0}^{\nu}\right)$ with $\theta_{0}=10$. The numeric solution of the Cauchy problem for (13) was integrated by the Runge-Kutta fourth-order method with step 0.01 . The trajectory near the stable slowly varying equilibrium is shown in the left panel. The parameters of (13) are $\nu=1 / 4, q=1 / 3, h=1$. The trajectory near the unstable equilibrium is shown in the right panel. The parameters of (13) are $\nu=3 / 4, q=1 / 16, h=1$. 
One can consider this system as a system of equations in closed form if one rewrites the variable $\theta$ in terms of the new variable $S$ :

$$
\frac{d S}{d \theta}=\theta^{\nu} / 2, \quad \theta=\frac{2}{2-\nu}\left(\frac{2-\nu}{2}\right)^{\frac{2}{2-\nu}} S^{\frac{2}{2-\nu}} .
$$

The formula for $\theta$ of $S$ looks too large, therefore, we will use $\theta$ and keep in mind that it is a function on $S$.

We rewrite the nonautonomous system (17) as a perturbed equation for a pendulum. For this we differentiate on $S$ the first equation using the second equation of the system. As a result, we get a differential second-order equation. Then we replace $r(S)$ by the root of the algebraic third-order equation for $r$ :

$$
r=-\frac{1}{2} \frac{d \alpha}{d S}-\frac{1}{2} \theta^{-\nu / 2}\left(r^{2}+\frac{h \cos (\alpha)}{1+\theta^{-\nu / 2} r}\right) .
$$

The asymptotic behavior of the smallest root for $\theta \rightarrow \infty$ has the form

$$
r \sim-\frac{\frac{d}{d S} \alpha}{2}-\frac{\left(\frac{d}{d S} \alpha\right)^{2}+4 h \cos (\alpha)}{8 \theta^{\frac{\nu}{2}}}+\frac{-\left(\frac{d}{d S} \alpha\right)^{3}-8 h \cos (\alpha)\left(\frac{d}{d S} \alpha\right)}{16 \theta^{\nu}} .
$$

The full form of the equation in the form of a perturbed pendulum, which is obtained from (17), is too lengthy. Therefore, we obtain an asymptotic form of this equation and keep such terms, which are important for our study as $\theta \rightarrow \infty$.

$$
\begin{aligned}
\frac{d^{2} \alpha}{d S^{2}} \sim 2 h \sin (\alpha)+2 q \theta^{\nu-1}-\frac{1}{2} \theta^{\frac{\nu}{2}-1}(4 q-\nu) \frac{d \alpha}{d S} \\
-\theta^{-1} 2 h q \cos (\alpha)-\theta^{-\frac{\nu}{2}-1} h q \cos (\alpha)\left(\frac{d \alpha}{d S}\right) .
\end{aligned}
$$

On bounded intervals of $S$ the function $\alpha$ is close to a solution of the equation for an unperturbed pendulum. In particular, the slowly varying asymptotic expansions are close to equilibria of an unperturbed pendulum constructed in Section 3.1.

When the phase space of Eq. (18) is projected onto the plane $(\alpha,(d \alpha) /(d S))$, the slowly varying solution near point $(0,0)$ looks like a saddle due to the structural stability of the saddle under perturbations.

The slowly varying solution near point $(\pi, 0)$ looks like a center. But it is well-known that the center is unstable under perturbations and can be transformed into a focus. Therefore, we should study the properties of the solutions near the slowly varying equilibrium which is close to $(\pi, 0)$.

Let us consider the energy of the unperturbed pendulum:

$$
E=\frac{\left(\alpha^{\prime}\right)^{2}}{2}+2 h \cos (\alpha)
$$

This term for the perturbed equation is a function on $S$. Let us derive the derivative of this function under perturbation (18):

$$
\begin{aligned}
\frac{d E}{d S} & \sim 2 q \theta^{\nu-1} \frac{d \alpha}{d S}-\frac{1}{2} \theta^{\frac{\nu}{2}-1}(4 q-\nu)\left(\frac{d \alpha}{d S}\right)^{2} \\
& -\theta^{-1} 2 h q \cos (\alpha) \frac{d \alpha}{d S}-\theta^{-\frac{\nu}{2}-1} h q \cos (\alpha)\left(\frac{d \alpha}{d S}\right)^{2} .
\end{aligned}
$$


The change of $E$ over one oscillation is

$$
\oint d E \sim-\frac{1}{2} \theta^{\frac{\nu}{2}-1}(4 q-\nu) \oint\left(\frac{d \alpha}{d S}\right) d \alpha .
$$

So the properties of $E$ under the oscillation of $\alpha$ are defined by the sign of $4 q-\nu$. In the terms of parameters for Eq. (8) we obtain the condition $a+b \neq 0$. Thus, we have the following theorem.

Theorem 3. If $a+b \neq 0$, then $E$ changes under one oscillation. The change is defined by the area inside the curve on the plane $(\alpha,(d \alpha) /(d S))$.

Corollary. If $a+b>0$, then the slowly varying equilibrium near $(\pi, 0)$ on the plane $(\alpha,(d \alpha) /(d S))$ is stable.

\subsection{Interval of validity for the asymptotic approximation}

Equation (7) was derived for a small amplitude of the solution in the special form (5):

$$
\left|\sqrt[3]{\epsilon} A e^{i(t-s)}\right| \ll 1
$$

Here we give an estimate for the interval of validity for such an asymptotic approach using the behaviors of amplitude $A$ in (5).

The asymptotic approach in the previous subsections shows that $|A| \sim \tau^{a}$. Let us substitute this formula into the asymptotic inequality (19). This yields

$$
\sqrt[3]{\epsilon} \tau^{a} \ll 1 .
$$

This leads to an estimate for the slow time variable $\tau$ :

$$
\tau \ll \epsilon^{-1 /(3 a)}, \quad \text { where } a>0 .
$$

This estimate shows the validity of the developed asymptotic approach for $\tau \rightarrow \infty$ as $\epsilon \rightarrow 0$.

\section{A phase locking and dephasing of trajectories}

One can divide the trajectories of the system (17) into resonant trajectories and a nonresonant trajectory. Such trajectories can be obtained by numerically using the Runge-Kutta method for the Cauchy problem with different parameters for the large independent variable $S$. In particular, Fig. 3 shows results of such numerical solutions. There are three trajectories with close behavior far from saddle. But near the saddle the nonresonant trajectory with the largest energy goes through a loop. The nonresonant trajectory with the lowest energy makes a turn and the third trajectory is captured into the resonance area inside the loop.

Depending on the parameters for the system of equations (17), one can not only obtain trajectories captured into the resonance area, but also see trajectories which leave the resonant area. Such trajectories correspond to dephasing. One can see such a trajectory in Fig. 4.

Let us consider the behavior of solutions to (18) near slowly varying equilibria which looks like a saddle on the plane $(\alpha,(d \alpha) /(d S))$. One can consider Eq. (18) as an equation for a pendulum with small momentum $M$ :

$$
\frac{d^{2} \alpha}{d S^{2}}=2 h \sin (\alpha)+M
$$

Here the momentum is: $M \sim 2 q \theta^{\nu-1}$ as $\theta \rightarrow \infty$. 

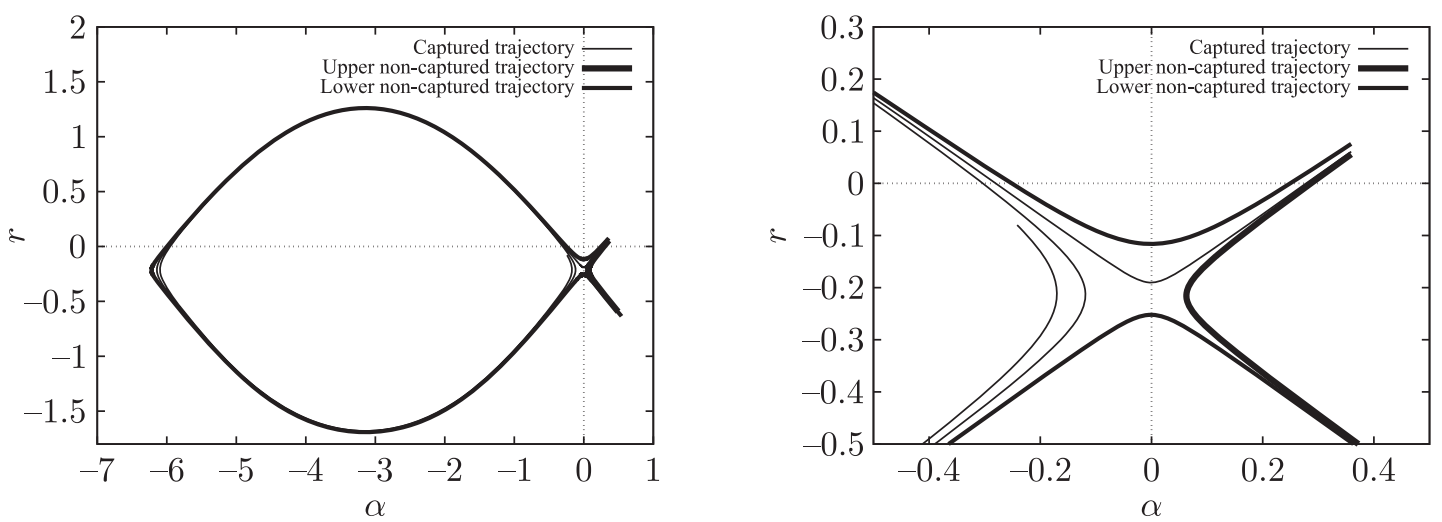

Fig. 3. Here we show projections onto the plane $\alpha, r$ for trajectories of the system (17), which are obtained by the Runge - Kutta fourth-order method with step 0.01 . The parameter values for the system are $h=1$, $q=1 / 3, \nu=1 / 4$. For the captured trajectory the solution was obtained by the Cauchy problem in the inverse direction of $S$ from $S=992$ to $S=1020$ and the Cauchy data were: $S=992, \alpha=-\pi+2.9$, $r=-0.08$. For nonresonant trajectories the solutions were obtained by solving the Cauchy problems for the positive direction by $S$. We obtain the solution with the largest energy on the interval $S \in[992,1010]$ with the Cauchy data $r=0.0815, \alpha=1.505, S=992$. For the solution with the smallest energy we obtain on the interval $S \in[992,998]$ with the Cauchy data: $r=0.0810, \alpha=1.505, S=992$.
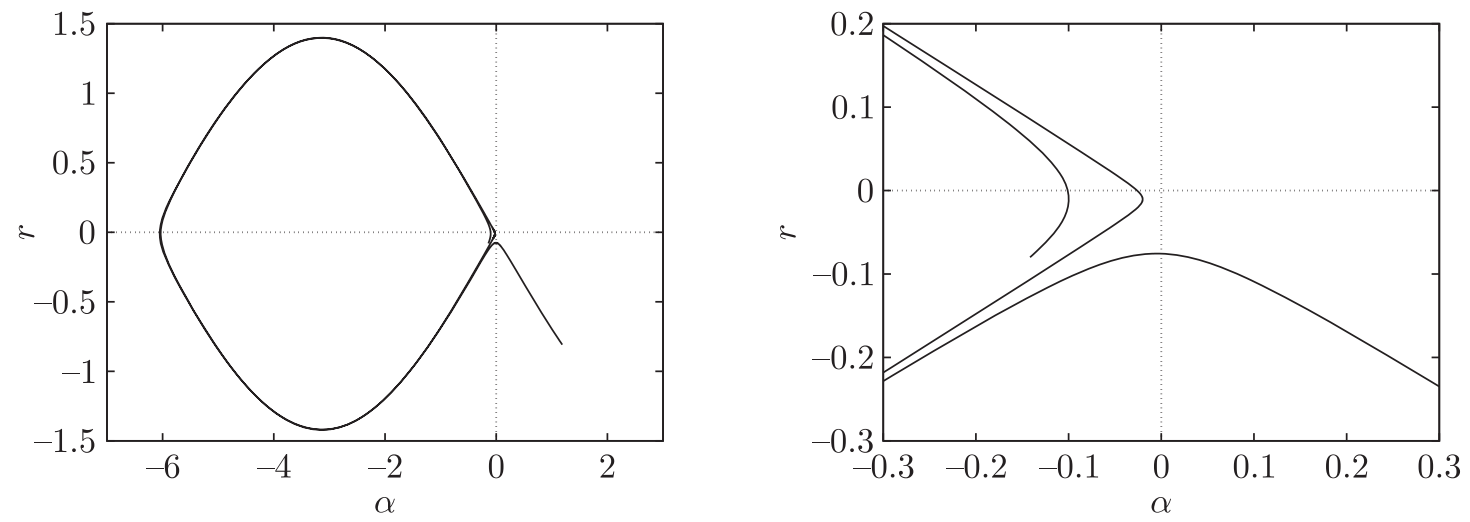

Fig. 4. Here one can see the projection onto the plane $\alpha, r$ of trajectories for the system (17). The trajectories were obtained by the Runge-Kutta fourth-order method with step 0.01 . The parameters values for the system are $h=1, q=1 / 16, \nu=3 / 4$. This trajectory leaves the resonant area. This shows the so-called dephasing. The solution was obtained on the interval $S \in[992,1020]$ with the Cauchy data: $S=992, \alpha=-\pi+3.0, r=-0.08$.

The theory of nonlinear resonance far from an equilibrium was developed by Chirikov in $[27,28]$. In the approach developed in [28] the nonlinear resonance is defined by the equation for a pendulum with an external torque.

Since the work [29] there has been a large body of research where a pendulum-like equation with additional torque as a constant in the leading-order term and the small perturbations of different kinds is derived (see [20, 23, 30-32]). These works are concerned with nonlinear resonances for oscillations of order $O(1)$.

For analysis of solutions of order $O\left(\epsilon^{1 / 3}\right)$ near the equilibrium there exists an older approach which leads to an equation with cubic nonlinearity like Eq. (4), see, for example, [17]. In this work we derive a pendulum-like equation from the equation for nonlinear resonance of order $O\left(\epsilon^{1 / 3}\right)$ 
as an asymptotic limit. The asymptotic relation between two of these approaches can be found in [33]. As a result, we obtain the pendulum equations with a special form of the torque which depends on time instead of the small perturbation parameter as in the cited references.

The equation with constant external momentum is integrated by quadrature. In the general case the equation with external momentum is not integrable. However, in our case the dependence on time is slow with respect to the oscillation frequency. Formally this gives an opportunity to consider the equation with slowly varying momentum as a perturbation of the equation with constant momentum.

There are three types of separatrices of an unperturbed pendulum with constant momentum. Firstly, there are homoclinic loops which begin and end in saddles. The other two types are unbounded separatrices which end or start from a saddle. According to Melnikov's theory, the centers and homoclinics are destroyed under a perturbation [34]. As a result, instead of the separatrix loops one obtains a curve emanating from a saddle and evolving to a focus or otherwise.

The trajectories which are captured into resonance pass through the gap between two separatrices close to the saddle, see $[33,35]$.

An important parameter for the pendulum is the energy:

$$
E=\frac{\left(\alpha^{\prime}\right)^{2}}{2}+h \cos (\alpha)-M \alpha
$$

The change of the energy near the saddle point defines the separatrix gap, which appears due to the perturbation [34]. For equation (18) the value of the gap between two separatrices is asymptotically close to the change of energy over one oscillation near the separatrix loop of the unperturbed pendulum:

$$
\Delta E \sim-\frac{1}{2} \theta^{\frac{\nu}{2}-1}(4 q-\nu) \oint_{\mathcal{L}}\left(\frac{d \alpha}{d S}\right) d \alpha .
$$

Here the curve $\mathcal{L}$ is a separatrix loop for $(20)$ when $M=2 q \theta^{\nu-1}$. The integral defines an area inside of this loop. Due to a small value of the external torque this square is asymptotically close to the square of the oscillation region of phase space for the pendulum when $M \equiv 0$. Then we obtain

$$
\oint_{\mathcal{L}}\left(\frac{d \alpha}{d S}\right) d \alpha \sim 4 \sqrt{h} \int_{0}^{2 \pi} \sqrt{1-\cos (\alpha)} d \alpha .
$$

The direct calculation shows

$$
\oint_{\mathcal{L}}\left(\frac{d \alpha}{d S}\right) d \alpha \sim 16 \sqrt{2 h} .
$$

This means that the gap between the separatrices is

$$
\Delta E \sim 8 \theta^{\frac{\nu}{2}-1}(\nu-4 q) \sqrt{2 h} .
$$

If $\nu-4 q \neq 0$, then the trajectories are captured into the resonance when $\nu-4 q<0$, otherwise the trajectories leave the resonance area when $\nu-4 q>0$. Therefore, as $\nu-4 q>0$, the trajectories can be seen to leave the resonance area. 
If one rewrites the values of parameters $q$ and $\nu$ using $a$ and $b$ from the primary equation (4), then one obtains the following theorem.

Theorem 4. Let the parameters $a$ and $b$ of (4) be such that $a+b \neq 0$. If $a+b>0$, then there exist trajectories which are captured into the resonance. If $a+b<0$, then there exist trajectories which leave the resonance area.

\section{Asymptotic behavior of measure for autoresonant solutions}

One of the important questions for applications of this asymptotic theory is as follows. How many trajectories from some area of initial data will be captured into autoresonance during a certain interval of time? In other words, which is the measure of captured trajectories?

The importance of the measure in the problems of nonlinear resonance was shown in [13], where the measure was used to obtain the probability of the capture into the resonance. The same probabilistic view on the capture was developed for a system with varying coefficients in [23]. Here we give calculations not for the resonant equation, but for the measure of the captured trajectories for the initial equation of the perturbed nonlinear oscillator.

Below we calculate the measure of trajectories of (2) which are captured into autoresonance. These calculations use the constructions of Sections 1 and 4. In particular, the constructed asymptotics are related to the nonlinear oscillator under external pumping by the following formulas:

$$
\begin{array}{r}
u(t, \epsilon) \sim \epsilon^{1 / 3} 2 \lambda \tau^{a}\left(1+\theta^{-\nu / 2} r\right) \exp (i \alpha) \exp (i(t-s))+c . c . \\
\frac{d}{d t} u(t, \epsilon) \sim \epsilon^{1 / 3} 2 i\left(1-\epsilon^{2 / 3} \lambda^{2} \tau^{2 a}\right) 2 \lambda \tau^{a}\left(1+\theta^{-\nu / 2} r\right) \exp (i \alpha) \exp (i(t-s))+c . c .
\end{array}
$$

The asymptotic behavior of the measure in the plane cross-section for a given $t$ of the phase space $\left(u, u_{t}, t\right)$ is

$$
\text { Mes }=\oint_{\mathcal{L}} d u_{t} \wedge d u \sim \epsilon^{2 / 3} 4 \lambda^{2} \tau^{2 a} \theta^{-\nu / 2} \oint_{\mathcal{L}} d r \wedge d \alpha \sim \epsilon^{2 / 3} 4 \lambda^{2} \tau^{2 a} \theta^{-\nu / 2} \oint_{\mathcal{L}} r d \alpha .
$$

The asymptotic behavior of the square inside the separatrix loop $\mathcal{L}$ is calculated in Section 4 . We need to make a change of variables from $\tau$ and $\theta$ to $t$ and $\epsilon$ :

$$
\begin{array}{r}
\text { Mes } \sim \epsilon^{2 / 3} 64 \lambda^{2} \tau^{2 a} \theta^{-\nu / 2} \sqrt{2 h}= \\
64 \epsilon^{2 / 3} \lambda^{2} \sqrt{2 f}(2 a+1)^{-\frac{3 a-b}{2(2 a+1)}} \lambda^{-\frac{2 b+3}{2(2 a+1)}} \times \\
\left(\epsilon^{2 / 3} t\right)^{2 a}\left(\frac{\lambda^{2}}{2 a+1}\left(\epsilon^{2 / 3} t\right)^{2 a+1}\right)^{-\frac{3 a-b}{4 a+2}} .
\end{array}
$$

After some calculations we obtain a formula for the asymptotic behavior of the measure, which depends on $t$ and $\varepsilon$ :

$$
M e s \sim 64 \epsilon^{(2+a+b) / 3} t^{(a+b) / 2} \sqrt{2 \lambda f} .
$$

\section{Conclusions}

In this work the following results have been obtained. First of all, we have found conditions for the chirp-rate and amplitude of the driver for autoresonance. There are $a>0$ and $a-1<b<3 a$. 
These conditions are necessary for autoresonance. Second, we have obtained conditions for the capture of the trajectory into autoresonance. There are additional conditions for the parameters of Eq. (4): $a-b>0$ and $a+b \neq 0$. The third result is the dephasing condition for the trajectory which leaves the resonance area: $a-b<0$ and $a+b \neq 0$. Also, one can see the measure of autoresonant asymptotics for the nonlinear oscillator $(21)$.

\section{References}

[1] Veksler, V.I., A New Method of Acceleration of Relativistic Particles, J. Phys. USSR, 1945, vol.9, pp. $153-158$.

[2] McMillan, E. M., The Synchrotron: A Proposed High Energy Particle Accelerator, Phys. Rev., 1945, vol. 68, nos. 5-6, pp. 143-144.

[3] Friedland, L., Spatial Autoresonance: Enhancement of Mode Conversion due to Nonlinear PhaseLocking, Phys. Fluids B, 1992, vol. 4, no. 10, pp. 3199-3209.

[4] Fajans, J. and Friedland, L., Autoresonant (Nonstationary) Excitation of Pendulums, Plutinos, Plasmas, and Other Nonlinear Oscillators, Am. J. Phys., 2001, vol. 69, no. 10, pp. 1096-1102.

[5] Uleysky, M. Yu., Sosedko, E. V., and Makarov, D. V., Autoresonant Cooling of Particles in Spatially Periodic Potentials, Tech. Phys. Lett., 2010, vol. 36, no. 12, pp. 1082-1084; see also: Pis'ma Zh. Tekh. Fiz., 2010, vol. 36, no. 23, pp. 31-38.

[6] Friedland, L., Autoresonance in Nonlinear Systems, Scholarpedia, 2009, vol. 4, no. 1, p. 5473.

[7] Glebov, S. G., Kiselev, O.M., and Lazarev, V.A., The Autoresonance Threshold in a System of Weakly Coupled Oscillators, Proc. Steklov Inst. Math. (Suppl.), 2007, vol. 259, suppl. 2, S111S123; see also: Tr. Inst. Mat. i Mekh. UrO RAN, 2007, vol. 13, no. 2, pp. 43-54.

[8] Kalyakin, L.A., Asymptotic Analysis of Autoresonance Models, Russian Math. Surveys, 2008, vol. 63, no. 5, pp. 791-857; see also: Uspekhi Mat. Nauk, 2008, vol.63, no. 5(383), pp. 3-72.

[9] Glebov, S. G., Kiselev, O.M., and Tarkhanov, N. N., Nonlinear Equations with Small Parameter: Vol. 1. Oscillations and Resonances, de Gruyter Ser. Nonlinear Anal. Appl., vol. 23/1, Berlin: De Gruyter, 2017.

[10] Kiselev, O. M., Introduction to Nonlinear Oscillations Theory, 2nd ed., Moscow: KomKniga, 2006.

[11] Kalyakin, L. A. and Sultanov, O. A., Stability of Autoresonance Models, Differ. Equ., 2013, vol. 49, no. 3, pp. 267-281; see also: Differ. Uravn., 2013, vol. 49, no. 3, pp. 279-293.

[12] Sultanov, O. A., Stability of Autoresonance Models Subject to Random Perturbations for Systems of Nonlinear Oscillation Equations, Comput. Math. Math. Phys., 2014, vol. 54, no. 1, pp. 59-73.

[13] Neishtadt, A. I., Passage through a Separatrix in a Resonance Problem with a Slowly-Varying Parameter, J. Appl. Math. Mech., 1975, vol. 39, no. 4, pp. 594-605; see also: Prikl. Mat. Mekh., 1975, vol. 39, nos. 4, pp. 621-632.

[14] Haberman, R., Nonlinear Transition Layers - the Second Painlevé Transcendent, Studies in Appl. Math., 1977, vol. 57, no. 3, pp. 247-270.

[15] Kiselev, O.M. and Glebov, S. G., An Asymptotic Solution Slowly Crossing the Separatrix near a Saddle-Centre Bifurcation Point, Nonlinearity, 2003, vol. 16, no. 1, pp. 327-362.

[16] Poincaré, H., Les méthodes nouvelles de la mécanique céleste: Vol. 3, Paris: Gauthier-Villars, 1899. (New York: Dover, 1957; reprint.)

[17] Krylov, N. M. and Bogolyubov, N. N., Introduction to Non-Linear Mechanics, Princeton: Princeton Univ. Press, 1949.

[18] Wasow, W. R., Asymptotic Expansion for Ordinary Differential Equations, Pure Appl. Math., vol. 14, New York: Interscience, 1965.

[19] Olver, F.W. J., Asymptotics and Special Functions. Computer Science and Applied Mathematics, New York: Acad. Press, 1974. 
[20] Arnol'd, V.I., Kozlov, V.V., and Neǐshtadt, A.I., Mathematical Aspects of Classical and Celestial Mechanics, 3rd ed., Encyclopaedia Math. Sci., vol. 3, Berlin: Springer, 2006.

[21] Neishtadt, A. I., Capture into Resonance and Scattering on Resonances in Two-Frequency Systems, Proc. Steklov Inst. Math., 2005, vol. 250, pp. 183-203; see also: Tr. Mat. Inst. Steklova, 2005, vol. 250, pp. 198-218.

[22] Treschev, D. and Zubelevich, O., Introduction to the Perturbation Theory of Hamiltonian Systems, Berlin: Springer, 2010.

[23] Neishtadt, A., Averaging Method for Systems with Separatrix Crossing, Nonlinearity, 2017, vol. 30, no. 7 , pp. 2871-2917.

[24] Friedland, L., Subharmonic Autoresonance, Phys. Rev. E, 2000, vol. 61, no. 4, pp. 3732-3735.

[25] Kalyakin, L. A., Asymptotic Solution of the Autoresonance Problem, Theoret. and Math. Phys., 2002, vol. 133, no. 3, pp. 1684-1691; see also: Teoret. Mat. Fiz., 2002, vol.133, no. 3, pp. 429-438.

[26] Garifullin, R. N., Asymptotic Analysis of a Subharmonic Autoresonance Model, Proc. Steklov Inst. Math. (Suppl.), 2003, suppl. 1, S75-S83; see also: Tr. Inst. Mat. i Mekh. UrO RAN, 2003, vol.9, no. 1 , pp. $56-63$.

[27] Chirikov, B. V., Resonance Processes in Magnetic Traps, J. Nucl. Energy: Part C, 1960, vol. 1, no. 4, pp. 253-260; see also: Soviet J. Atom. Energy, 1960, vol. 6, no. 6, pp. 464-470.

[28] Chirikov, B. V., A Universal Instability of Many-Dimensional Oscillator Systems, Phys. Rep., 1979, vol. 52, no. 5, pp. 264-379.

[29] Chirikov, B. V., Passage of Nonlinear Oscillatory System through Resonance, Sov. Phys. Dokl., 1959, vol. 4, pp. 390-394; see also: Dokl. Akad. Nauk SSSR, 1959, vol. 125, no. 5, pp. 1015-1018.

[30] Molchanov, A. M., The Resonant Structure of the Solar System, Icarus, 1968, vol. 8, nos. 1-3, pp.203215.

[31] Kevorkian, J., On a Model for Reentry Roll Resonance, SIAM J. Appl. Math., 1974, vol. 26, no. 3, pp. 638-669.

[32] Murdock, J., Qualitative Theory of Nonlinear Resonance by Averaging and Dynamical Systems Methods, in Dynamics Reported: Vol.1, U. Kirchgraber, H. O Walther (Eds.), Wiesbaden: Vieweg+Teubner, 1988, pp. 91-172.

[33] Kiselev, O.M. and Tarkhanov, N., The Capture of a Particle into Resonance at Potential Hole with Dissipative Perturbation, Chaos Solitons Fractals, 2014, vol. 58, pp. 27-39.

[34] Mel'nikov, V.K., On the Stability of the Center for Time Periodic Perturbations, Trans. Moscow Math. Soc., 1963, vol. 12, pp.1-57; see also: Tr. Mosk. Mat. Obs., 1963, vol. 12, pp. 3-52.

[35] Kiselev, O. and Tarkhanov, N., Scattering of Trajectories at a Separatrix under Autoresonance, J. Math. Phys., 2014, vol. 55, no. 6, 063502, 24 pp. 